\title{
Cambios en la relación médico-paciente y nivel de satisfacción de los médicos
}

\author{
M Luz Bascuñán $\mathbf{R}^{\mathbf{a}}$.
}

\section{Changes in physician-patient relationship and medical satisfaction}

Background: Physician-patient relationship is the basis of clinical practice. In the last decades, the changes in this relationship have influenced the degree of satisfaction of physicians with their practice. Aim: To explore the perception of physicians about changes in physician-patient relationship and its impact in their personal and professional satisfaction. Material and methods: Qualitative study involving 44 physicians from the Metropolitan Region of Chile. Focus groups and semi-structured interviews were used. Results: All physicians mentioned changes on physician-patient reltionship as one of the most important factors that has impact on their satisfaction. The main cause for the deterioration of this relationship is a generalized climate of distrust that leads to frustration and disenchantment with the profession. Conclusions: This feeling is in accordance with other reports showing that patients are progressively acquiring more protagonism in health care decisions. The reconstruction of the bond between patients physicians within these new scenarios is indispensable to improve the degree of satisfaction of both patients and professionals (Rev Méd Chile 2005; 133: 11-6). (Key-words: Ethics, medical; Physician-patient relations; Professional-patient relations)

Recibido el 6 de julio, 2004. Aceptado en versión corregida el 22 de septiembre, 2004. Departamento de Psiquiatría y Salud Mental, Facultad de Medicina, Sede Oriente. Universidad de Chile

apsicóloga, Master of Science (MSc)

L a práctica de la medicina ha cambiado en las últimas décadas. Se han realizado múltiples esfuerzos para describir la naturaleza de estos cambios, así como su impacto en la satisfacción de los usuarios. Sin embargo, menos atención se ha prestado a cómo los médicos experimentan y enfrentan los cambios en su profesión.

En números anteriores de esta revista se ha aludido a los cambios sociales de la práctica

Correspondencia a: M Luz Bascuñán R. Avenida Salvador 486, Providencia. Fono-Fax: 2748855.

E-mail: mbascunan@med.uchile.cl médica, a partir de un estudio que realizó el Departamento de Psiquiatría y Salud Mental Oriente de la Facultad de Medicina de la Universidad de Chile y el Departamento de Salud Pública de la Facultad de Medicina de la Pontificia Universidad Católica de Chile ${ }^{1}$. El presente trabajo se propone continuar esta reflexión centrándose en los cambios en la relación médico-paciente y su impacto en el grado de satisfacción de los médicos.

La relación clínica constituye una fuente crítica de satisfacción para los pacientes, así como una 
variable que distingue al grupo de médicos satisfechos e insatisfechos con su profesión ${ }^{2,3}$. La literatura muestra que la relación y comunicación médico paciente produce beneficios para la salud psicológica y física del paciente y aumenta su confianza y compromiso ${ }^{4-6}$. Así mismo, los problemas en esta relación son un factor crucial en la decisión para cambiar de médico o iniciar acciones legales contra los profesionales ${ }^{7}$.

Se han descrito distintos modelos de relación clínica en función de la distribución del poder y responsabilidad de médico y paciente. El tipo de relación que se establezca dependerá de las características del paciente y el tipo de situación clínica. Además, esta relación es dinámica y los roles que se asumen varían en el tiempo. Sin embargo, cualquiera sea el modelo que se adopte, el énfasis actual es puesto en el respeto y participación mutua de médico y paciente en la toma de decisiones sanitarias. Esto se asocia a una nueva visión de paciente, ahora capaz y autónomo, pese a estar enfermo, lo que a su vez genera un nuevo modelo de relación asistencial donde el paciente tiene un rol protagónico ${ }^{8}$.

El principio bioético que da soporte a esta nueva concepción es el de autonomía ${ }^{9}$. Si bien el reconocimiento de este principio impone límites al clásico modelo paternalista, esto no significa que la autonomía del paciente no tenga también límites, ni que se niegue la autonomía del profesional. Además, así como el rol protagónico de los pacientes no disgusta a todos los profesionales, tampoco gusta a todos los pacientes ${ }^{10,11}$.

Los cambios en la medicina y en la organización de los servicios de salud han generado un impacto en los médicos. Sin embargo, hasta la fecha no disponemos de estudios empíricos y sistemáticos que den cuenta de esta situación en nuestro medio. El objetivo del presente trabajo es explorar y describir la percepción que un grupo de médicos chilenos de la Región Metropolitana, en diversos sectores de práctica profesional, tienen sobre los cambios en la relación clínica y su impacto en el nivel de satisfacción profesional.

\section{MATERIAL Y MÉTODO}

Se realizó un estudio cualitativo incluyendo una muestra de 44 médicos de distintas edades y sectores de práctica profesional de la Región Metropolitana (Tabla 1). Se utilizó una metodología cualitativa, ya que ésta es especialmente adecuada para explorar descriptivamente un proceso de cambio. Este método descarta todo presupuesto acerca del tema a investigar y se propone observar cómo se da el fenómeno de interés de manera natural y desde la perspectiva de los informantes.

Se utilizaron dos métodos de recolección de datos, consistentes en la realización de tres grupos focales y la aplicación de una encuesta auto-

Tabla 1. M étodos de recolección de datos y muestra de sujetos incluidos en el estudio

\begin{tabular}{|c|c|c|c|}
\hline $\begin{array}{l}\text { Método de } \\
\text { recolección de datos }\end{array}$ & $\begin{array}{l}\text { Sector de práctica } \\
\text { profesional }\end{array}$ & $\mathrm{n}$ & Edad y sexo \\
\hline \multirow{3}{*}{$\begin{array}{l}\text { Cuestionario } \\
\text { Auto administrado }\end{array}$} & \multirow{2}{*}{$\begin{array}{l}\text { Sector Público Académicos } \\
\text { *(ENCI) }\end{array}$} & \multirow[t]{2}{*}{10} & \multirow{3}{*}{$\begin{array}{l}\text { Promedio }=61 \text { años } \\
8 \text { varones, } 2 \text { mujeres } \\
\text { Promedio }=38 \text { años } \\
2 \text { varones, } 5 \text { mujeres }\end{array}$} \\
\hline & & & \\
\hline & $\begin{array}{l}\text { Atención Primaria/Med. } \\
\text { Familiar *(ENC II) }\end{array}$ & 7 & \\
\hline \multirow{4}{*}{ Grupos Focales } & Sector público Académicos & 7 & \multirow{4}{*}{$\begin{array}{l}\text { Promedio }=60 \text { años } \\
5 \text { varones, } 2 \text { mujeres } \\
\text { Promedio }=50 \text { años } \\
6 \text { varones y } 1 \text { mujer } \\
\text { Promedio }=48 \text { años } \\
12 \text { varones, } 1 \text { mujer }\end{array}$} \\
\hline & $\begin{array}{l}*(\mathrm{GFI}) \\
\text { Sector Privado }\end{array}$ & 7 & \\
\hline & $*(\mathrm{GFII})$ & & \\
\hline & $\begin{array}{l}\text { Servicio de Urgencia } \\
\quad * \text { (GFIII) }\end{array}$ & 13 & \\
\hline
\end{tabular}

*Abreviación utilizada en la descripción de resultados. 
administrada sobre la base de las mismas tres preguntas básicas:

1. ¿Cuáles son los cambios principales que los médicos perciben en la práctica de la medicina durante el período en que han ejercido? 2. ¿Cuál es la percepción del impacto que tales cambios han tenido sobre el grado de satisfacción con la práctica? 3. ¿Cuáles han sido las principales estrategias de afrontamiento que los médicos refieren han aplicado frente a estos cambios?

Se realizó un análisis de contenido de los datos que fue sometido a una triangulación por el equipo interdisciplinario de investigación, constituido por cuatro médicos, una psicóloga, una socióloga y un metodólogo. Este análisis permitió determinar aquellas percepciones compartidas en la muestra estudiada, respecto a los principales factores de cambio y su efecto en el grado de satisfacción de los médicos (de acuerdo a la frecuencia e intensidad de los diferentes temas en el discurso de los participantes).

\section{Resultados}

Los grupos médicos estudiados enfatizaron y valoraron de diferente manera los cambios ocurridos en la medicina en los últimos años. Sin embargo, en todos ellos se mencionó la relación médico-paciente como uno de los cambios de mayor impacto en su satisfacción profesional y personal, y como un cambio valorado más negativa que positivamente. A continuación se distinguen las principales dimensiones de cambio de la relación clínica percibidas por los médicos participantes. Para ello se utilizan las palabras de los mismos médicos, con el objeto de evitar el sesgo interpretativo e ilustrar su lógica discursiva.

\section{Tecnificación y especialización de la medicina} Según la percepción de los médicos, la tecnificación y especialización médica han provocado en gran parte su deshumanización. También contribuye al «desmembramiento del paciente en órganos y a la pérdida de la visión de conjunto» (ENC I). En este sentido el «delanto tecnológico desvirtúa la vocación médica»(GFI). Así mismo la surocratización de la medicina se refleja en una progresiva despersonalización de la relación» (ENC I). «No hay tiempo para crear un lazo afectivo con el paciente» (GFIII). Los estándares internacionales, la medicina basada en la evidencia y la tecnología van reemplazando el arte de la medicina, la experiencia y habilidades clínicas del médico. «odo está programado; el número de pacientes, el tiempo para cada uno, lo que debe hacerse en cada caso»(GFI). El médico se ha ido transformado en eun funcionario que debe aplicar estos estándares y satisfacer las demandas de un cliente»(ENCI).

Introducción de variables económicas en la profesión La introducción de variables económicas en la profesión también es asociada a la pérdida del arte en medicina y al nivel de control en el ejercicio de la profesión. «El sistema médico se ha invadido por los gestores» (ENCII) y «a medicina comienza a transformarse en un mercado donde se maximiza la relación costo-efectividad y se presiona al médico a ser productivo» (GFIII). «Antes era más importante la calidad que la cantidad, ahora interesan tantos pacientes por hora como si fuera una fábrica de calcetines» (GFIII). «El usuario ya no es nuestra prioridad y de ahí nace nuestra desgracia»(ENCI).

Judicialización de la medicina

La creciente introducción del derecho en medicina interferinía en la tradicional relación uno a uno pues «el paciente está respaldado por otros (actores sociales)»(GFII) generando un tipo de medicina defensiva. «a sociedad y los pacientes están mucho más exigentes en reclamar responsabilidad legal a los médicos» (ENC I). Los médicos «deben cubrirse las espaldas»(GFI). «Empieza la medicina por el miedo, miedo de que todo lo del paciente caiga encima»(GFIII).

Cambios sociales y características de los usuarios Explosión y masificación del conocimiento: Los pacientes están más informados sobtienen datos en internet o en la prensa no especializada, lo que hace que incluso en muchas oportunidades cuenten con información mayor o más actualizada que las de los médicos» (ENCI). También «a tecnología ha llegado a conocimiento del paciente por lo tanto el paciente llega pidiendo exámenes» (GFII). «El mayor conocimiento aunque sea superficial... les da más seguridad a los pacientes»(GFI) y cambia el tipo de relación que se establece. 
Pérdida de respeto y deferencia: Existe una sensación de nostalgia «del reconocimiento y respeto de la sociedad y los pacientes» (GFI). «a relación médico-paciente se ha aplanado y se cuestiona lo que el médico hace, se pierde el respeto de los pacientes»(GFI). La mayor «ngratitud y agresividad de la gente hace que sea otra cosa»(GFIII). «Eso del -gracias Dr. usted me ayudó- es lo que satisface y hoy no es así, se debe hacer lo que tiene que hacer y pobre si no lo hace». (GFIII).

Redefinición de expectativa: Se percibe un cambio fundamental y masivo en el nivel de exigencia y expectativas del paciente y la sociedad» (GFI). «Se cae en el juego de responder a las expectativas... y uno pide y pide exámenes, este es un globo que tiene que reventar»(GFII). La medicina se encarece pero «si no se hacen todos esos exámenes la gente esta insatisfecha»(GFI). Muchas expectativas son poco realistas. Los avances en medicina no pueden evitar acontecimientos naturales de la vida como el envejecimiento, el sufrimiento y la muerte. « $\mathrm{El}$ público ha cambiado y siente derecho a exigir a estar sano... nos exigen que no se mueran» (GFIII). Por un lado «ya no somos dioses, pero sí somos dioses porque no podemos equivocarnos ni fallar» (GFIII).

Los cambios descritos son asociados a un clima de creciente desconfianza generando un importante impacto en el nivel de satisfacción del médico (Figura 1).
El problema es la «desconfianza... se supone que todo lo malo que pasa es por una mala praxis o porque existe un inconfesado interés económico detrás»(GFI). «Hay una sensación de estar en guardia por el cuestionamiento y desconfianza de pacientes»(GFII). En este contexto se reporta un desencanto con la profesión. «a mayoría estudió la carrera porque nos gustaba y creíamos que podíamos ser útiles, pero se enfrenta una realidad distinta»(GFIII). «Existe la vocación de dar pero no hay quien la reciba»(GFIII). «Produce frustración que los pacientes se sientan insatisfechos a pesar de los esfuerzos que uno ha hecho» (GFI).

El vínculo con el paciente aparece también como un factor central para afrontar los cambios. Resguardar este vínculo constituińa entonces un mecanismo de protección ante la frustración experimentada. «engo la esperanza que una medicina humana y racional me conserve en contacto con mis pacientes» (ENCI). Se enriquece enormemente «a vida personal viendo la retribución afectiva de una buena relación médico paciente» (ENCI). «Mis estrategias personales (ante los cambios) han sido revisar los fundamentos del que hacer y buscar espacios para recrear las bases de una práctica más satisfactoria con los pacientes»(ENCI).

En general, los cambios en la medicina y en la relación clínica tienden a ser percibidos como heterónomos más que autónomos. Esto es, como eventos más determinados por factores externos que no guardan relación con la propia voluntad ni la acción individual. «Hay que bailar al son que nos

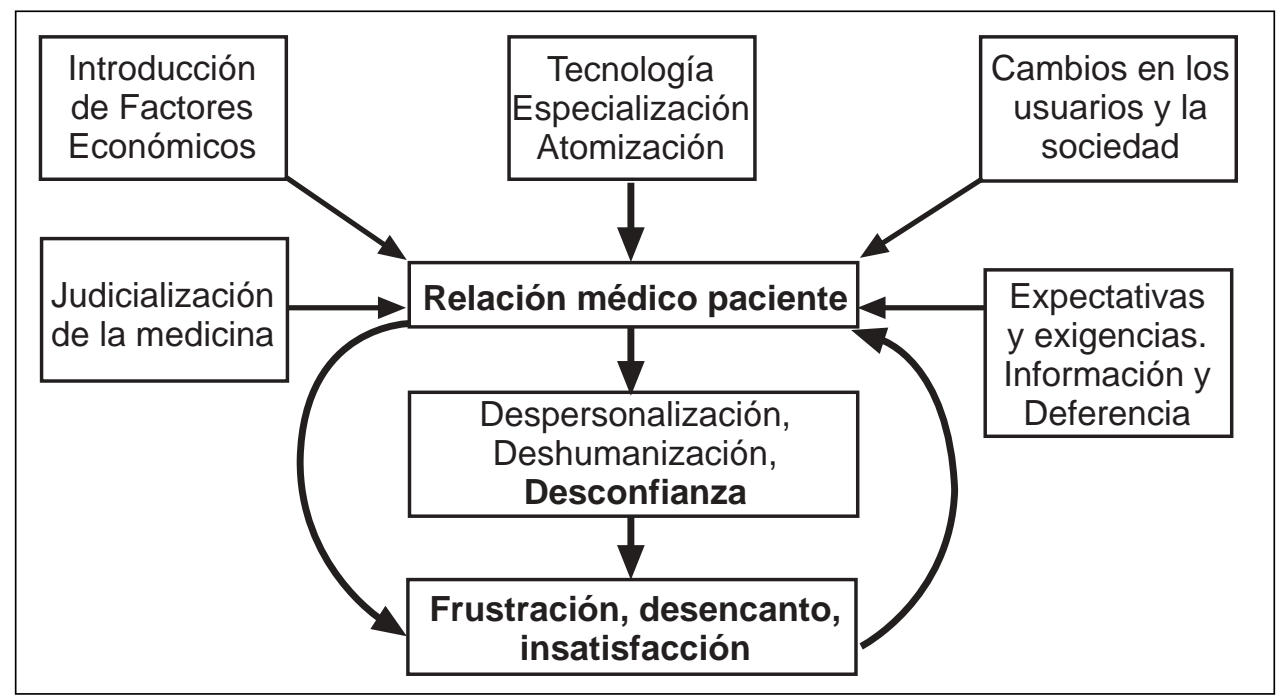

FiguRA 1. Dimensiones de cambio e impacto percibido en la relación médico-paciente. 
pongan... debiera promoverse este tipo de discusión para participar, tener presencia y que se escuche nuestra voz»(GFI). Se observa entonces la necesidad de los médicos de expresar su malestar y de disponer de espacios de reflexión e intercambio de experiencias. «sto (grupo focal) es como una catarsis... rara vez viene alguien a preguntarnos cómo nos sentimos» «No hay canales de comunicación para solucionar nuestros problemas»(GFII).

\section{DisCUSIÓN}

Los datos obtenidos en este estudio son consistentes con la literatura internacional, en cuanto reflejan la percepción de cambios en la relación clínica, donde la participación del paciente es cada vez más activa. Sin embargo, más que percibir las conveniencias de estos cambios, ellos tendieron a ser negativamente evaluados.

Los cambios en la relación clínica implican, en primer lugar, un nuevo tipo de profesionalismo con un balance diferente del poder y la autonomía del profesional y paciente. En este contexto, la insatisfacción profesional tiene relación con la percepción de una invasión en la profesión, donde el médico va perdiendo progresivamente el control sobre su práctica, mientras los usuarios y otras agencias sociales la van ganando. La creciente participación de los pacientes, también enfrenta a los profesionales a reconocer la incertidumbre y producir frustración en quienes creen en la certeza definitiva de la medicina. Para algunos, médicos y pacientes han contribuido a la idealización de la medicina mediante un pacto irrealista que finalmente provoca frustración en ambos ${ }^{12}$. Es necesario reemplazar este trato por uno más realista, donde los médicos acepten sus limitaciones y los pacientes ajusten sus expectativas y conozcan la naturaleza incierta de la medicina. La confianza es la base de la relación médicopaciente y de acuerdo a este estudio, ésta es la que finalmente y por diversos motivos es amenazada. El reconocimiento que los pacientes tienen nuevos medios de acceder a la información y a utilizar métodos alternativos de tratamiento, requiere de nuevas habilidades, donde más que ofrecer un «saber», los médicos deben evaluar la información y entregar la mejor alternativa según los valores y preferencias del paciente.
Existiná un cambio en la noción de sbuen médico» y buen paciente». Esta parece ser la era de la medicina que toma la perspectiva del paciente en las decisiones y transformación del sistema de salud. Se proclama un paciente informado, capaz, empoderado. El buen paciente del futuro trae lista de preguntas y espera respuestas precisas, sabe qué puede exigir y se sabe respaldado, sabe cuán involucrado desea estar en las decisiones, posee acceso y uso libre de su ficha médica, solicita opiniones adicionales y usa diferentes medios de comunicación con el médico ${ }^{13}$. En otras palabras, el buen paciente del futuro es actualmente un paciente difícil.

Nos enfrentamos a una realidad compleja que requiere distintos niveles de análisis. Si bien se describe a un paciente más informado, autónomo y exigente, la idea de un paciente como individuo que decide en conciencia aisladamente es una abstracción. El papel del médico, su ponderación y su rol de consejero es insustituible.

Pese a la variedad de actividades que un médico puede desarrollar actualmente, un motivo central para escoger la profesión, sigue siendo el interés de relacionarse y atender personas. La relación con los pacientes también explicaría que la generación de médicos mayores sobreviva a las dificultades. La frustración de los médicos generada por los cambios en la relación clínica, se entiende entonces, como expresión de un proceso de adaptación que resalta la relevancia de este vínculo para el ejercicio satisfactorio de la profesión.

Como se refleja en los resultados, los cambios en la medicina y en la relación clínica, tienden a ser vistos como externos a los médicos más que como el resultado de un proceso en el que se ha tenido una participación activa. Es relevante examinar más profundamente este fenómeno, si consideramos que la actitud ante los cambios, así como su impacto subjetivo, están fuertemente determinados por el nivel de participación y compromiso (personal y colectivo) con el proceso de cambio social.

El presente estudio describe la percepción compartida y testimonial de los médicos participantes. Sin embargo, sus resultados también indican la necesidad de proseguir esta línea de investigación, con una muestra masiva y representativa de médicos, que permita realizar distinciones según la edad, género, especialidad y lugar de práctica profesional entre otras variables, dando cuenta de la diversidad y complejidad de la profesión médica actual. 


\section{REFERENCIAS}

1. Horwitz N. El cambio en la práctica médica. Desafíos psicosociales para la profesión. Rev Méd Chile 2004; 132: 768-72.

2. Zuger A. Dissatisfaction with medical practice. $\mathrm{N}$ Engl J Med 2004; 350: 69-75.

3. ReAmes HR y Dunstone DC. Professional satisfaction of physicians. Arch Intern Med 1989; 149: 1951-6.

4. LLYd M, Bor R. Communication skills for medicine. Ed. Churchill Livingston, Londres, 2001.

5. Little P, Everitt H, Wiwamson I, Warner G, Gould C ET AL. Observational study of effect of patient centredness and positive approach on outcomes of general practice consultations. BMJ 2001; 323: 908-11.

6. Beck RS, Daughtridge R, Sloane PD. Physicianpatient communication in the primary care office: A Systematic Review. J Am Board Fam Pract 2002; 15: 25-38.

7. Vincent C, Young M, Phiwips A. Why do people sue doctors? A study of patients and relatives taking legal action. Lancet 1994; 25; 343(8913): 1609-13.
8. Simón P, GutiérREz J. Bioética para médicos. Med Clín 2001; 17: 99-106.

9. GaLÁn CoRTÉs JC. La responsabilidad médica y el Consentimiento informado. Rev Méd Urug 1999; 15: 5-12.

10. BeAuchamp TL, FADEN RR 1. History/Meaning and Elements of Informed Consent. En: Reich T, Ed. Encyclopedia of Bioethics. USA: MacMillan Library, 1995.

11. Kaplan SH, Greenfield S, Gandek B, Rogers WH, WARE JE. Characteristics of physicians with participatory decision-making styles. Ann Intern Med 1996; 124: 497-50.

12. Sмiтн R. Why are doctors so unhappy? There are probably many causes, some of them deep. BJM 2001; 322: 1073-4.

13. JADAD A, Riso C, ENKIN M. I am a good patient, believe it or not. BMJ 2003; 326: 1293-5.

\section{Agradecimientos}

Deseamos agradecer a todos los médicos que tuvieron la gentileza de participar en este estudio expresando sus opiniones y compartiendo sus experiencias personales. 